\title{
Static form-finding of normal and defective catenaries based on the analytical exact solution of the tensile Euler-Bernoulli beam
}

Proc IMechE Part F:

J Rail and Rapid Transit

2019, Vol. 233(7) 69I-700

(C) IMechE 2018

Article reuse guidelines:

sagepub.com/journals-permissions

DOI: 10.1 I77/09544097/8808990

journals.sagepub.com/home/pif

@SAGE

\author{
Farzad Vesali', Mohammad Ali Rezvani' (1), \\ Habibolah Molatefi' (1) and Markus Hecht ${ }^{2}$
}

\begin{abstract}
The aim of this research is to propose and develop an analytical exact solution for finding the static equilibrium configuration of a catenary before and after incurring defects such as tension loss or a broken dropper. The procedure includes considering the steady-state solution of the dynamic motion equation of the contact wire and the messenger cable. The wire and the cable are considered as tensile Euler-Bernoulli beams. The stiffness matrix of the beam is configured and is used to calculate the dropper's dead load. Progressively, a novel method is proposed to find the equilibrium configuration of the same catenary after the defect. The results prove that the tension loss in the messenger cable is more precarious than the tension loss in the contact wire. The broken dropper causes a significant sag in the subspan and increases the static forces of the adjacent droppers. A comparison with field measurements justifies the accuracy of the results of the proposed model.
\end{abstract}

\section{Keywords}

Catenary, static form-finding, broken dropper, tension loss, exact solution

Date received: 8 April 2018; accepted: 12 September 2018

\section{Introduction}

\section{Background}

Most of the high-speed trains collect electrical power from the overhead catenary system. Therefore, the dynamic interaction between the pantograph and the catenary should be studied in order to analyze stable current collection and to ensure a good contact quality. Since the static initial condition of the catenary including the shape of the contact wire and the messenger cable has significant role on the interaction between the pantograph and the catenary system, any practical model needs to estimate the initial shape of the contact wire and the messenger cable.

\section{Formulation of the problem of interest for this investigation}

Several models are available for simulating the dynamic interaction of the pantograph and the catenary. ${ }^{1}$ Facchinetti and Bruni ${ }^{2}$ stated that any method to determine the static position of the catenary needs to include some key modeling issues in the pantograph and catenary interaction. Additionally, the literature proves that among the many methods available for the calculation of the static force of droppers, the outcomes are not the same. The differences in the results are considerable. ${ }^{1}$ A comparison between the static forces $(\mathrm{N})$ of droppers that are calculated using various finite element model (FEM) or finite differences method (FDM) software programs proves a high standard deviation of force of each dropper between the results. Therefore, the aim of this research is to develop an accurate, fast method for calculating the static forces of droppers for the catenary systems.

\footnotetext{
'School of Railway Engineering, Iran University of Science and Technology, Tehran, Iran

${ }^{2}$ Department of Rail Vehicle, Technical University of Berlin, Berlin, Germany
}

\section{Corresponding author:}

Mohammad Ali Rezvani, Iran University of Science and Technology, Narmak, Tehran I6846-13II, Iran.

Email: rezvani_ma@iust.ac.ir 


\section{Literature survey}

PantoCat ${ }^{3}$ is a model that uses an optimization technique to minimize the function that quantifies the distance between the static deformed geometry of the contact wire and its specified position. SPOPS ${ }^{4}$ uses an iterative method to find the length of each dropper and the initial configuration of the catenary. $\mathrm{PCaDA}^{4}$ uses an iterative procedure in which a nonlinear optimization is used in each step to drive the catenary geometry and tension in the contact wire and the messenger cable. The above-mentioned software and other software perform simulations that are based on FEM or FDM. Therefore, it makes sense when the iterative or the optimization methods are used in these software in order to calculate the static form of the catenary. There are several definitions for form-finding or estimation of the initial equilibrium state of a form-active structure. In other words, it is a method of finding an optimal shape of a structure that is in a state of static equilibrium. ${ }^{5}$ The existing form-finding methods for discrete networks, such as the force density method, dynamic relaxation, updated reference strategy and a variety of other methods, are discussed and compared. ${ }^{6}$ In the overhead catenary system, the length of the dropper should be adjusted in such a way that the contact wire sets in a planned profile. In fact, when dealing with laying the catenaries, the shape of one element in the assembly is defined and the shape of the other elements in addition to the interaction forces needs to be calculated. In this field, it is then rather natural to calculate the initial equilibrium configuration of the railway catenary. For example, an accurate, robust and fast method based on analytical catenary equations for a twodimensional form-finding problem was previously proposed by Lopez-Garcia et al. ${ }^{7}$ Such et al. ${ }^{8}$ extended this method to three-dimensional tension cable structures. In addition, the geometry variation method, ${ }^{9}$ the separated model method, ${ }^{10}$ the negative sag method, ${ }^{11}$ the optimization methods on constraint problems, ${ }^{12}$ the minimal error of the dropper tension, ${ }^{13}$ minimizing the distance between the static deformed geometry of the contact wire and its specified position ${ }^{3}$ are able to evaluate the static configuration of the catenary. Recently, Yang et al. ${ }^{14}$ proposed a dynamic equilibrium method to compute the initial equilibrium configuration of the railway catenary. The initial configuration of the catenary is used not only to simulate the dynamic reaction of the pantograph and catenary ${ }^{15}$ but it can also be used to make geometric optimization on the catenary parameters. ${ }^{16}$ In addition, the exact solution of the static configuration of a defective catenary is useful to monitor the catenary by pantograph, i.e. when it is possible to simulate the effect of any defect on pantograph acceleration, it would be possible to measure the pantograph acceleration and estimate the type of defect in the catenary.

\section{Contribution of this study}

Prior studies have attempted to calculate the static force of droppers in the catenary systems with noticeable low accuracies. However, this study proposes not only the exact solution for calculating the static force of droppers but also the method for evaluating the static form of catenary after three types of defects, including tension loss in messenger cable, tension loss in contact wire and the breaking of one dropper.

\section{Organization of this article}

Primarily, the analytical model of the catenary is developed, and the stiffness matrix of the contact wire is calculated. This is required in order to find the forces in the droppers. Configuration of the contact wire and the messenger cable is then generated by relying on the steady-state solution of the dynamic equation of the tensile beam. Therefore, according to the classification by Veenendaal and Block, ${ }^{6}$ the method that is presented in this research can be considered as a combination of the stiffness matrix method and the dynamic equilibrium method. Then, the calculated force of each dropper is used for evaluating the static form of the catenary after defect.

\section{Description of the catenary model}

This research proposes a two-dimensional analytical exact solution for form-finding of catenaries. To serve the purpose, the following questions need to be worked out:

Q1: What is the size of the force in each dropper that can set the contact wire on its proper configuration (Figure 1(a))? To elaborate further, when the long tensile beam is considered as the model of the contact wire and it is possible to apply force in location of dropper connection points, what is the best force distribution to put the contact wire in a demanded profile? Should it be distributed uniformly, or its distribution depends on the distance between the droppers?

Q2: What will be the configuration of the contact wire and the messenger cable after applying forces of the droppers? Clearly, after applying the calculated forces of the droppers, the configuration of the contact wire should be the same as in the demanded profile.

Q3: What will be the new equilibrium shape of the contact wire and the messenger cable after experiencing the defects such as the loss of tension of the contact wire or the messenger cable? i.e. what would happen if one dropper breaks (Figure 1(b)).

In Figure 1, $T_{m c}$ and $T_{c w}$ are the tension of the messenger cable and the contact wire, respectively. $k_{s}$ and $k_{d r}$ are the stiffnesses of the support and the 


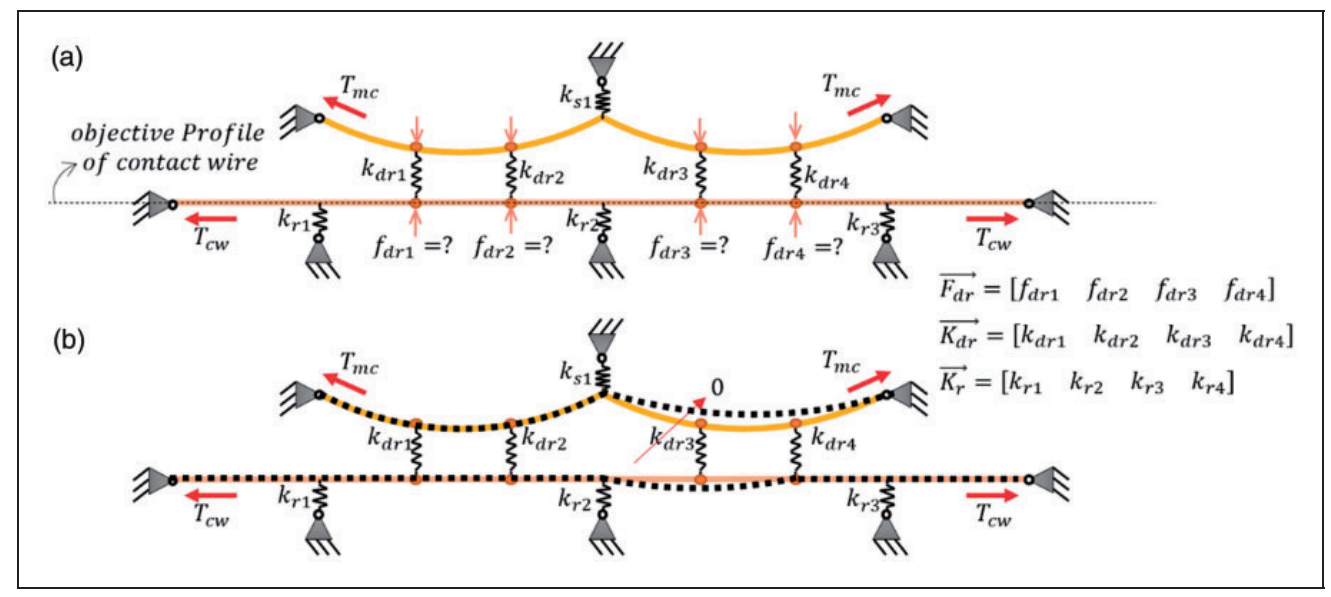

Figure I. (a) The basis for calculating dropper forces to set the contact wire on its objective profile. (b) The basis for recalculating the equilibrium configuration of the catenary after the dropper breakage and tension variation.

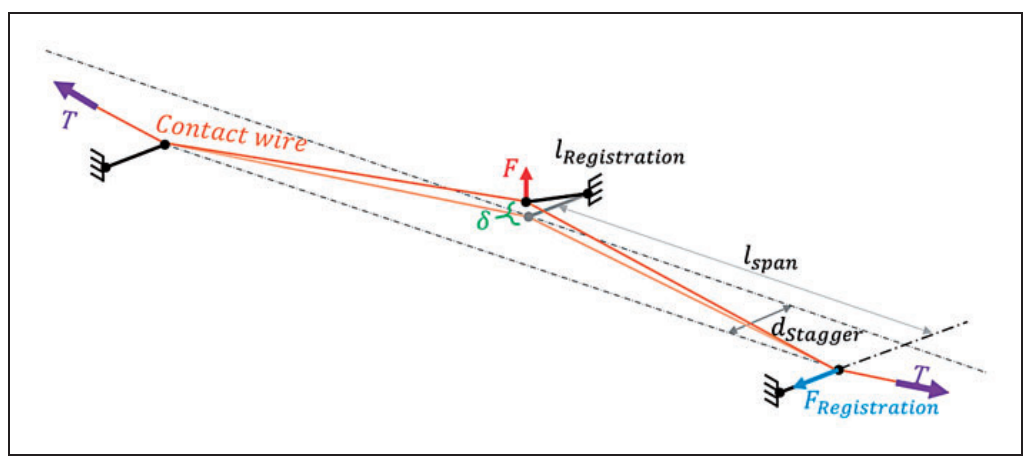

Figure 2. The vertical stiffness of the registration arm and the zigzag in the contact wire.

registration arm. $k_{d r i}$ is the stiffness of the $i$ th dropper. $f_{d r i}$ is the static force or the dead load of the $i$ th dropper that will be calculated in this research.

For the modeling purposes, the messenger cable and the contact wire are considered within the tensile Euler-Bernoulli beam theory. The droppers are considered as linear springs. The dropper clamps and the registration arms are considered as concentrated masses. The vertical stiffness of the registration arm is a function of the tension of the contact wire $\left(T_{c w}\right)$, length of the stager $\left(d_{\text {stagger }}\right)$, length of the registration $\operatorname{arm}\left(l_{\text {Registration }}\right)$ and span length $\left(l_{\text {span }}\right)$, as given in equation (1)

$$
k_{r}=2 T_{c w} \times\left(\frac{d_{\text {stagger }}}{l_{\text {Registration }} \times l_{\text {span }}}+\frac{1}{l_{\text {span }}}\right)
$$

The parameters that are used in equation (1) are presented in Figure 2 and the definitions are given in the Appendix.

The properties for the contact wire and the messenger cable that are schematically shown in Figure 1 are presented in Table 1. These properties are extracted from the standard document EN 50318, 2002.

\section{Formulation and the mathematic model}

Both the contact wire and the messenger cable are considered as tensile beams and the droppers are modeled as nonlinear springs with significant stiffness in tension and zero stiffness in compression. Since all droppers in the static form of the catenary are under tension, the nonlinearity of the droppers can be ignored in the static form-finding. The differential equation governing the tensile beam can be described according to equation (2), and the related boundary condition compatible with the modeling that is used in Figure 1 is presented in equation (3). The boundary condition contains zero deflection and momentum in supports

$$
\begin{gathered}
\rho A w_{, t t}+C w_{, t}-T w_{, x x}+E I w_{, x x x x}=F_{g}+F_{d}(x), \quad 0 \leqslant x \leqslant l, t \geqslant 0 \\
w(0, t)=0, \quad w(l, t)=0, \quad w_{, x x}(0, t)=0, \quad w_{, x x}(l, t)=0
\end{gathered}
$$

In the above equations, $w(x, t)$ is the beam transverse displacement in time $t$ and space $x, \rho A$ is the mass per unit length of the beam, $C$ is the beam 
Table I. Properties of the contact wire (CW) and the messenger cable (MC). ${ }^{17}$

\begin{tabular}{|c|c|c|c|c|c|c|c|c|}
\hline Parameter & Value & Parameter & Value & \multicolumn{4}{|c|}{ Parameter } & Value \\
\hline Span length (m) & 60 & Mass/unit length of CW $(\mathrm{kg} / \mathrm{m})$ & 1.35 & \multicolumn{4}{|c|}{ Mass/unit length of $M C(\mathrm{~kg} / \mathrm{m})$} & 1.07 \\
\hline Encumbrance $(\mathrm{m})$ & 1.2 & Tension of $\mathrm{CW}(\mathrm{kN})$ & 20 & \multicolumn{4}{|c|}{ Tension of MC (kN) } & 16 \\
\hline Pre-sag at mid-span (mm) & 0 & Bending stiffness of $\mathrm{CW}\left(\mathrm{N} \mathrm{m}^{2}\right)$ & 195.0 & \multicolumn{4}{|c|}{ Bending stiffness of $\mathrm{MC}\left(\mathrm{N} \mathrm{m}^{2}\right)$} & 131.7 \\
\hline Stagger $(\mathrm{mm})$ & \pm 200 & Mass of dropper clamps on CW (g) & 0 & \multicolumn{4}{|c|}{ Mass of dropper clamps on MC (g) } & 0 \\
\hline No. of spans & 10 & Mass of stager $(\mathrm{g})$ & 400 & \multicolumn{4}{|c|}{ No. of droppers per span } & 9 \\
\hline Stiffness of dropper $(\mathrm{kN} / \mathrm{m})$ & 10 & Stiffness of registration arm $(\mathrm{N} / \mathrm{m})$ & 340 & \multicolumn{4}{|c|}{ Mass of droppers $(\mathrm{kg})$} & 0 \\
\hline Dropper position in each spa & $\operatorname{an}(\mathrm{m})$ & 10.5 & 17 & 23.5 & 30 & 36.5 & 49.5 & 55 \\
\hline
\end{tabular}

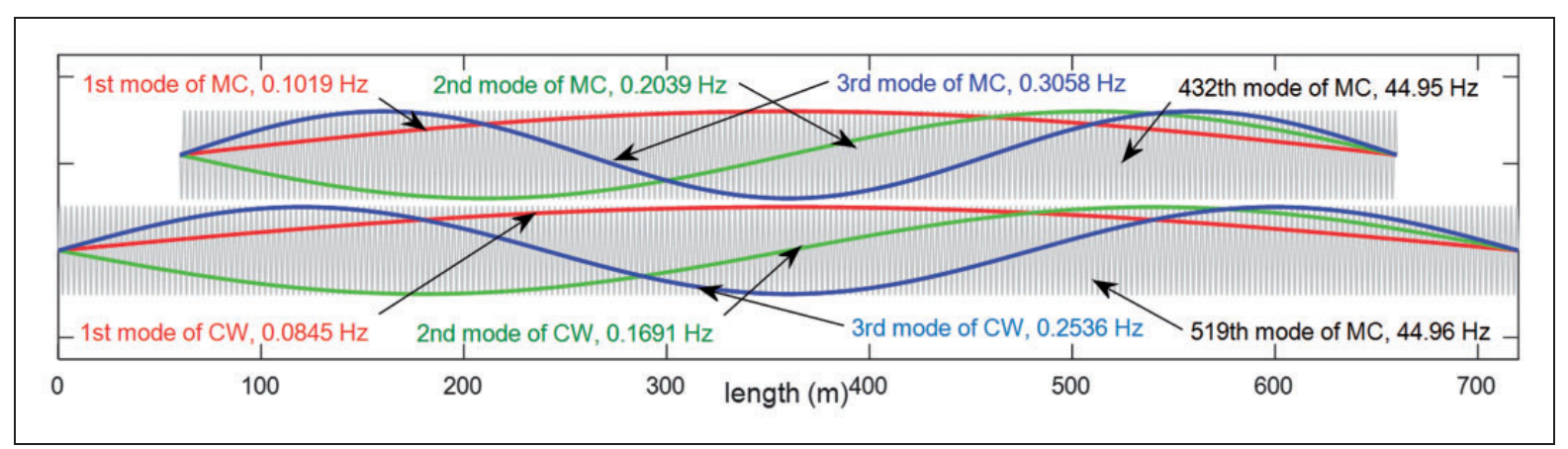

Figure 3. The first three and the last mode shapes and natural frequencies of the messenger cable (MC) and the contact wire (CW) that are considered in the model.

damping coefficient, $T$ is the axial load applied to the beam that is positive in tension and $E I$ is the beam flexure rigidity. The gravity load $\left(F_{g}\right)$ and the concentrated force exerted on the droppers or the registration $\operatorname{arm}\left(F_{d}\right)$ are also involved on the right-hand side of the equation. According to the method of the separation of variables, the deflection function of the beam can be described as in equation (4)

$$
w(x, t)=\sum^{\varphi_{i}}(x) q_{i}(t)
$$

In equation (4), $q_{i}(t)$ is the Rayleigh quotient or the temporal term and $\varphi_{i}(x)$ is the spatial term or the mode shape function of the $i$ th mode shape. By considering the proper boundary conditions, the modal analysis of the beam can be accomplished, and the natural frequencies and mode shapes of the beam can be extracted. In equation (2), it is noticed that the droppers' and the support forces appear in the left-hand side of the equation; therefore, they are not considered in the mode shapes and natural frequencies of the contact wire and the messenger cable. Figure 3 presents the first, second, third and the last mode shapes and the related natural frequencies of both the messenger cable and the contact wire.

The final form of the catenary is configured based on coupling the two individual beams (messenger cable and contact wire) via droppers. In fact, the mode shapes and the natural frequencies of the final form of the catenary are like the ones presented in Figure 3 because the dropper forces and the support forces are considered as excitation forces at the lefthand side of the differential equation.

Expansion of the eigenfunctions can be used in order to change the partial equations of a tensile beam to a system of ordinary deferential equation (equations (5) to (7))

$$
\ddot{q}_{i}(t)+\xi_{i} \dot{q}_{i}(t)+\omega_{i}^{2} q_{i}(t)=\alpha_{n} \int_{0}^{l} \varphi_{i}(x)\left(F_{g}+F_{d}\right) \mathrm{d} x, \quad t>0
$$

$$
\begin{aligned}
& \alpha_{n}=\frac{1}{\rho A \int_{0}^{l} \varphi_{i}^{2}(x) \mathrm{d} x} \\
& \xi_{i}=\frac{C}{\rho A}
\end{aligned}
$$

If the excitation forces on the right-hand side of equation (5) are caused by a spring, damper or a mass, they will shift to the left-hand side of the equation and add to the coefficients of $q_{i}(t), \dot{q}_{i}(t)$ and $\ddot{q}_{i}(t)$.

In static form-finding, $\dot{q}_{i}{ }^{(t)}$ and $\ddot{q}_{i}{ }^{(t)}$ are zero and $q_{i}(t)$ can be calculated according to equations (8) and (9)

$$
\begin{aligned}
& q_{i}(t)=\beta_{n} \int_{0}^{l} \varphi_{i}(x)\left(F_{g}+F_{d}\right) \mathrm{d} x, \quad t>0 \\
& \beta_{n}=\frac{1}{\omega_{i}^{2} \rho A \int_{0}^{l} \varphi_{i}^{2}(x) \mathrm{d} x}
\end{aligned}
$$


When $q_{i}(t)$ is calculated, the deflection of the tensile beam (the messenger cable or the contact wire) can be calculated using equation (4).

\section{Calculating the droppers' dead load}

Calculations for the static equilibrium configuration of the contact wire and the messenger cable after incurring some common damage to the catenary, such as the dropper break or tension loss in the contact wire or the messenger cable, are exercised in this section.

The static shape of the contact wire has a major role on the dynamic interaction of the pantograph and catenary. In catenary design, it is mostly common to keep the contact wire in a straight line while in some designs, a small pre-sag is considered. ${ }^{9}$ In analytical simulation, the static shape of the contact wire is determined by calculating the droppers' dead load. For the purposes of this research, the droppers' dead load is calculated using the vector form of the linear spring formula (equations (10) and (11))

$$
\begin{aligned}
& {\overrightarrow{F_{d r}} n d \times 1}=\left[K_{c w}\right]_{n d \times n d} \times \overrightarrow{\Delta w_{c w n}} \times 1 \times 1 \\
& {\overrightarrow{\Delta w_{c w n d \times 1}}}=\left(\overrightarrow{w_{g c w}}-\overrightarrow{w o_{c w}}\right)_{n d \times 1}
\end{aligned}
$$

The properties related to the contact wire and the messenger cable are denoted by subscripts $c w$ and $m c$, respectively. In equation (10), nd is the total number of the droppers in the section, $\overrightarrow{F_{d r}}$ is the droppers' dead load, $K_{c w}$ is the contact stiffness matrix. $\overrightarrow{w_{g c w}}$ is the vertical deflection of the contact wire due to the gravity at dropper connection points and $\overrightarrow{w o_{c w}}$ is the objective profile of the contact wire at the dropper connection points. In order to calculate the stiffness matrix of the contact wire, a unique load is applied to the droppers' connection points and the deflection of the contact wire is calculated based on equations (4) and (8). The vertical stiffness of the registration arm ( $k_{r}$ in equation (1) and Figure 4) is considered in the calculation of the stiffness matrix. $w_{2,1}$ is the vertical deflection of the connection point of the second dropper due to the application of a unique load at the connection point of the first dropper. The stiffness matrix of the contact wire is calculated using equation (12)

$$
\left[K_{c w}\right]=\left[\begin{array}{ccc}
w_{1,1} & w_{1,2} & \ldots w_{1,3} \\
w_{2,1} & w_{2,2} & \ldots \\
\vdots & \vdots & \vdots \\
w_{n d, 1} & w_{n d, 2} & \ldots w_{n d, n d}
\end{array}\right]^{-1}
$$

The force vector of the droppers can be calculated by inserting $K_{c w}$ from equation (12) into equation (10). Then, the shape of the contact wire and the messenger cable can be achieved by applying this force in upward and downward directions, respectively. Figure 5 presents the shape of the contact wire and the messenger cable after applying the calculated dead load of the droppers. The particulates of the catenary for this example are according to the EN 50318 standard documents.

In Figure 5(b), it is shown that applying the calculated dropper forces can set the contact wire on its objective profile. The force distribution is almost uniform and the droppers close to the support should carry higher dead load in order to set the contact wire in a straight horizontal line.

\section{Static form-finding of a defective catenary}

A further aim of this study is form-finding of defective catenaries. Tension loss in the messenger cable and in the contact wire and the breaking of droppers are considered as three common defects that can happen in catenaries. A simple configuration of a catenary by considering spring and mass components is presented in Figure 6.

In Figure 6, $\left[K_{m c}\right]$ and $\left[K_{c w}\right]$ represent the stiffness matrix of the messenger cable and the contact wire, respectively. The procedures for calculating the elements of these matrices are described in the prior sections of this article. $\left[K_{d r}\right]$ is a matrix that contains the stiffness of each dropper on its main diagonal elements (equation (13)). $\overrightarrow{M_{m}}$ and $\overrightarrow{M_{c}}$ are the vectors that

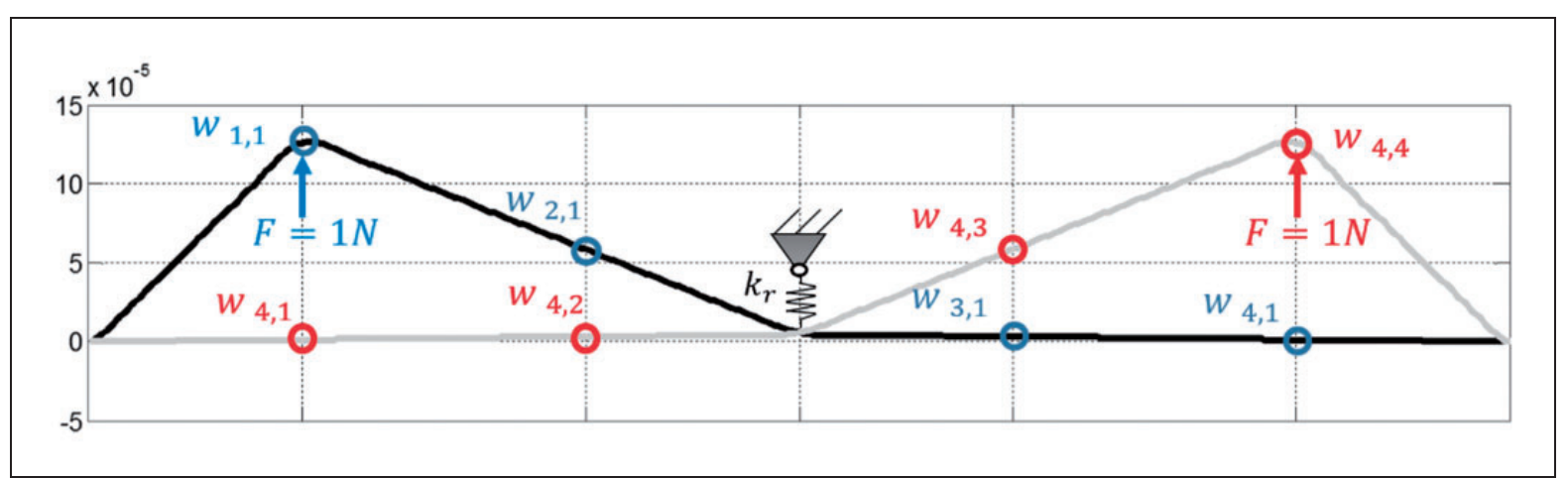

Figure 4. The schematic for applying a unique load on the contact wire for calculating the stiffness matrix. 
(a)

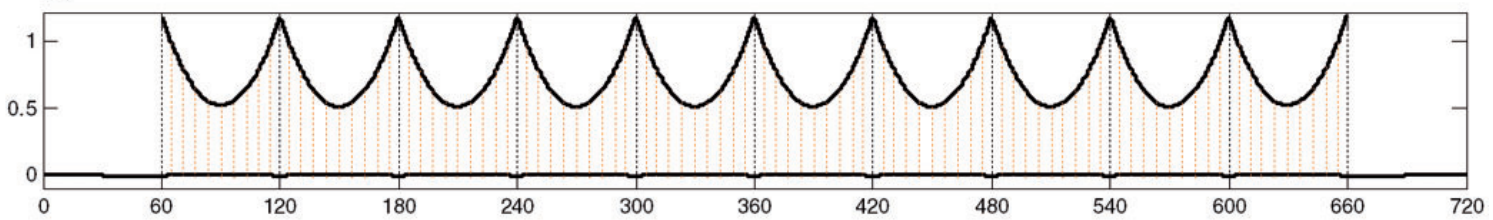

(b)

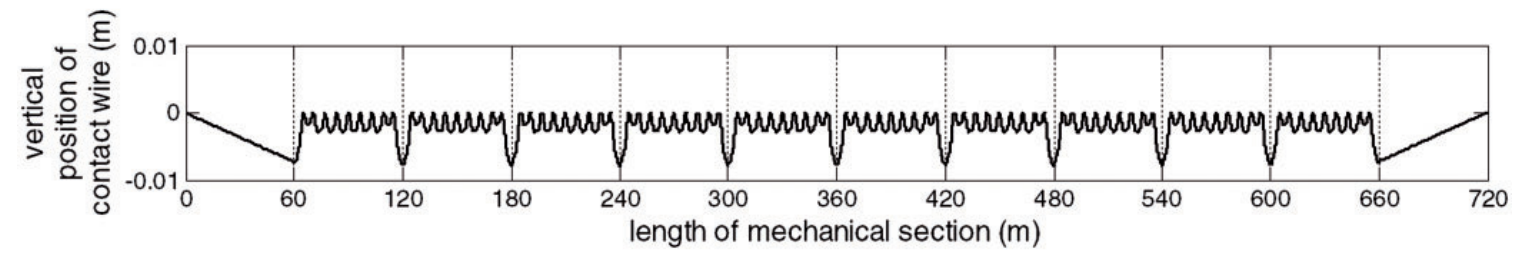

(c)

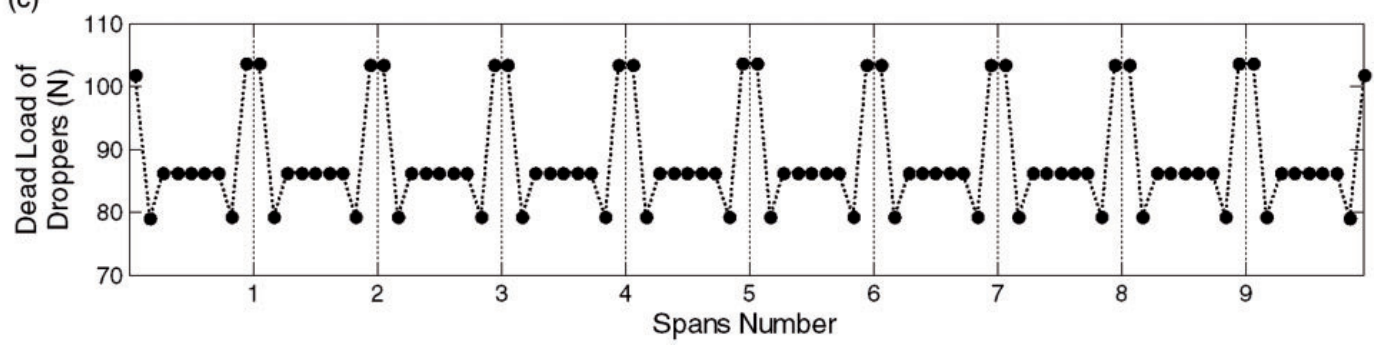

Figure 5. (a) Static shape of the catenary after applying the calculated dead load of the droppers, (b) equilibrium configuration of the contact wire and (c) static force distribution of the droppers.

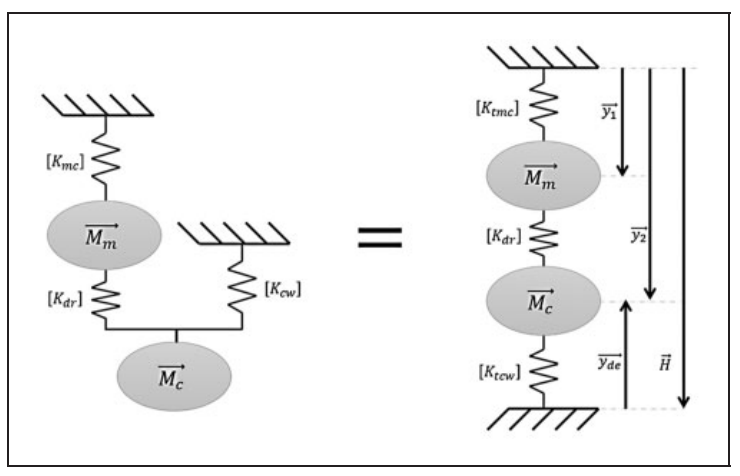

Figure 6. A simple model of a catenary by considering the stiffness matrix and the mass matrix.

describe the equivalent mass of the messenger cable and the contact wire (in addition to all attached masses such as the clamp masses, etc.) at connection points of the droppers for the messenger cable and the contact wire, respectively. In order to achieve the equivalent mass of the messenger cable and the contact wire, the stiffness matrix and the vertical deflection due to the gravity are needed (equations (14) and (15)). In Figure $6, \overrightarrow{y_{1}}$ is a vector that contains the vertical deflection of the dropper connection points on the messenger cable measured from the reference axes of the messenger cable, $\overrightarrow{y_{2}}$ is a vector that contains the vertical deflection of dropper connection points on the contact wire measured from the reference axes of the messenger cable. $\overrightarrow{y_{o}}$ is a vector that contains the objective position of the dropper connection points on the contact wire measured from the reference axes of the contact wire (in a straight contact wire without pre-sag, $\overrightarrow{y_{o}}=0$ ). $\vec{H}$ in Figure 6 is a vector that does not change due to the defect and describes the offset between the contact wire and the messenger cable. $\vec{H}$ acts as the main hint to find the equilibrium configuration of the catenary after the defect. $\overrightarrow{y_{1}}, \overrightarrow{y_{2}}$ and $\overrightarrow{y_{o}}$ are presented in Figure 1(a)

$$
\begin{aligned}
& {\left[K_{d r}\right]=\left[\begin{array}{cccc}
k_{d r} & 0 & 0 & 0 \\
0 & k_{d r} & 0 & 0 \\
0 & 0 & k_{d r} & 0 \\
0 & 0 & 0 & k_{d r}
\end{array}\right]} \\
& \overrightarrow{M_{m}}=\left[K_{m c}\right] \times \overrightarrow{w_{g m c}} \\
& \overrightarrow{M_{c}}=\left[K_{c w}\right] \times \overrightarrow{w_{g c w}}
\end{aligned}
$$

The contents of vector $\vec{H}$ should be calculated in the catenary before considering the defect. For this purpose, the system of equation (16) should be solved with $\overrightarrow{y_{1}}$ and $\overrightarrow{y_{2}}$ as the unknown variables

$$
\begin{aligned}
& {\left[K_{m c}\right] \overrightarrow{y_{1}}=\overrightarrow{M_{m}}+\overrightarrow{M_{c}}+\left[K_{c w}\right] \overrightarrow{y_{d e}}} \\
& {\left[K_{d r}\right] \overrightarrow{y_{1}}+\left[K_{d r}\right] \overrightarrow{y_{2}}=\overrightarrow{M_{c}}+\left[K_{c w}\right] \overrightarrow{y_{d e}}}
\end{aligned}
$$


$\vec{H}$ can be calculated from equation (18)

$$
\vec{H}=\overrightarrow{y_{2}}+\overrightarrow{y_{d e}}
$$

The hint is that $\vec{H}$ will be constant before and after any defect. In a defective catenary, depending on the type of the defect, $\left[K_{d r}\right],\left[K_{c w}\right]$ and $\left[K_{m c}\right]$ can change. For example, in the case of a broken dropper, $\left[K_{d r}\right]$ will change and one array of it turns to zero. When there is a tension loss in the messenger cable or the contact wire, the corresponding stiffness matrix will change. It should be noted that by changing the stiffness matrix, the equivalent mass should be recalculated according to equations (14) and (15). In equilibrium equation of a defective catenary, the variables $\overrightarrow{y_{1}}, \overrightarrow{y_{2}}$ and $\overrightarrow{y_{d e}}$ are unknown. However, since $\vec{H}$ is calculated previously, the system of equations (19) and (20) can be derived

$$
\begin{aligned}
& {\left[K_{m c}+K_{d r}^{\prime}\right] \overrightarrow{y_{1}^{\prime}}-\left[K_{d r}^{\prime}\right] \overrightarrow{y_{2}^{\prime}}=\overrightarrow{M_{m}}} \\
& -\left[K_{d r}^{\prime}\right] \overrightarrow{y_{1}^{\prime}}+\left[K_{d r}^{\prime}+K_{c w}\right] \overrightarrow{y_{2}^{\prime}}=\overrightarrow{M_{c}}+\left[K_{c w}\right] \vec{H}
\end{aligned}
$$

In these equations, it is assumed that one dropper is broken and $\left[K_{d r}\right]$ has changed to $\left[K_{d r}^{\prime}\right] \cdot \overrightarrow{y_{1}^{\prime}}$ and $\overrightarrow{y_{2}^{\prime}}$ are the position of the droppers' connection points on the messenger cable and the contact wire after the defect, respectively

$$
\overrightarrow{F_{d r}}=\left[K_{d r}^{\prime}\right]\left(\overrightarrow{y_{2}^{\prime}}-\overrightarrow{y_{1}^{\prime}}\right)
$$

By calculating $\overrightarrow{y_{1}^{\prime}}$ and $\overrightarrow{y_{2}^{\prime}}$, the force vector of the droppers $\left(F_{d r}^{\prime}\right)$ can be calculated according to equation (21). The shape of the contact wire and the messenger cable can be achieved by applying these forces in the upward and downward directions, respectively.

\section{Results}

As an example, a mechanical section that includes 10 spans is considered. The properties of the catenary are the same as in Table 1. Primarily, the effect of having a broken dropper at the middle of the second span is studied.

Figure 7 presents the equilibrium configuration of a catenary before and after sustaining a defect of missing one dropper at the middle of the second span. The zoomed area in this figure shows that when a dropper breaks, the messenger cable rises up $22 \mathrm{~mm}$ and the contact wire sags $13 \mathrm{~mm}$ at the location of the broken dropper. The sag between the two droppers in this catenary before the defect was $2.8 \mathrm{~mm}$, and the sag under registration arm was $7.8 \mathrm{~mm}$. In addition, the forces from the adjacent dropper to the broken dropper increased from $86 \mathrm{~N}$ to $130 \mathrm{~N}$ (around $50 \%$ ).

The model that is used to draw the results in Figure 7 is for a catenary system that includes 10 spans while each span contains nine droppers. It comprises $360 \mathrm{~m}$ of a contact wire and $300 \mathrm{~m}$ of a messenger cable. The dropper forces that are calculated for this model exhibit symmetry for the whole model along its mid-point. This means that as an example the outcome for the second span in this model is as symmetric as the eighth span. However, if the model includes nine spans, the fifth span will behave symmetrically along its mid-point.

In the next step, the effect of $5 \%$ tension loss in the messenger cable is studied.

The data shown in Figure 8 demonstrate that by $5 \%$ reduction in the tension of the messenger cable, the mid-point of the messenger cable and the contact wire in the second span sags for $20 \mathrm{~mm}$. The forces of the droppers close to the end of the span increase, but the forces of the droppers located at the middle of the span decrease.

As the last step, the effect of $10 \%$ reduction in the tension in the contact wire is studied.

The effect of $10 \%$ reduction in the tension in the contact wire is illustrated in Figure 9. This set of data presents that changing the position of the messenger cable due to this defect is negligible. The sag in the contact wire at the position of the registration arm increases from $7.7 \mathrm{~mm}$ to $8.5 \mathrm{~mm}$. Therefore, the

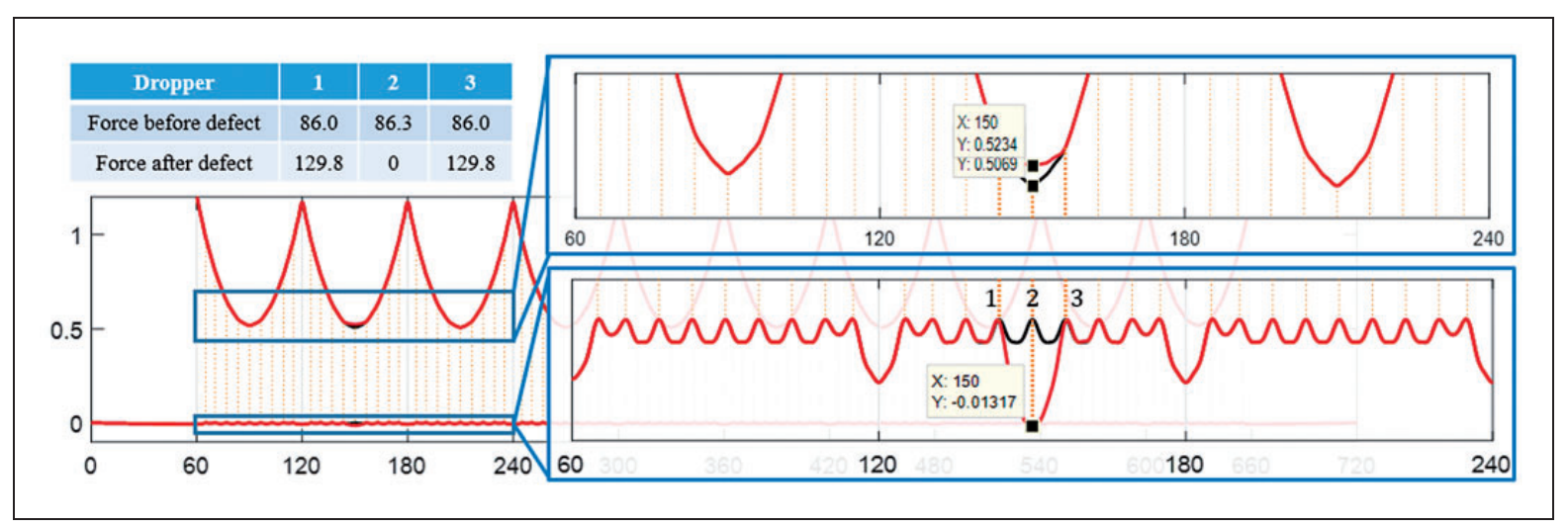

Figure 7. The effect of breaking one dropper at the middle of the second span on the shape of the contact wire and the messenger cable and the force on adjacent droppers (the black (dark) line: before the defect; the red (grey) line: after the defect). 


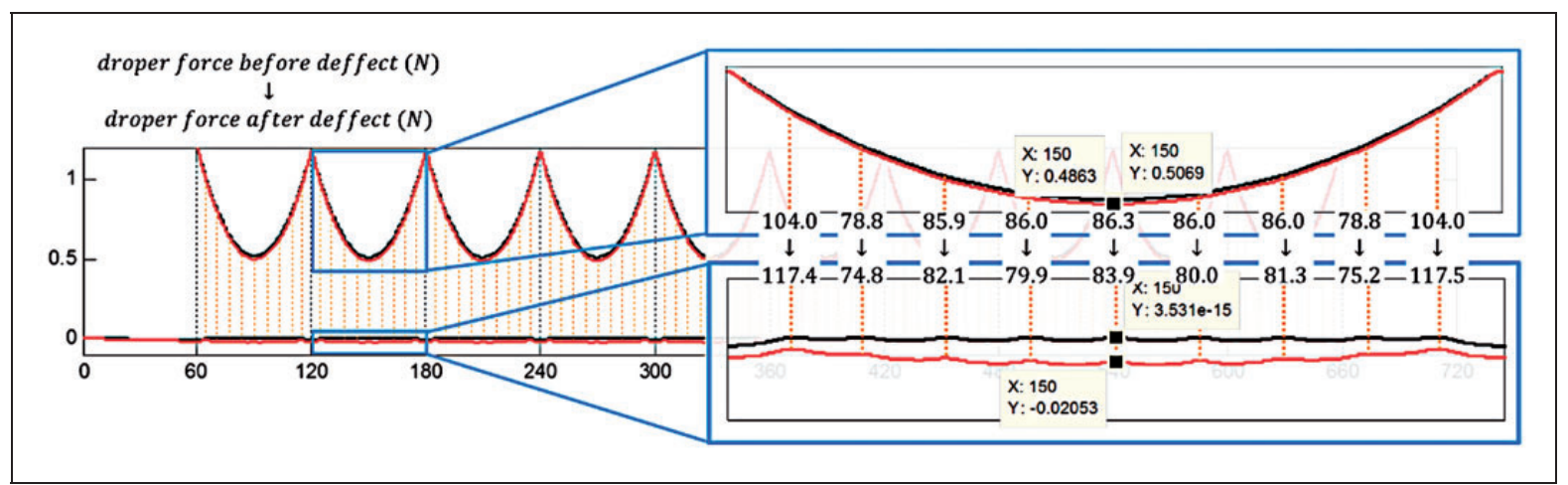

Figure 8. The effect of $5 \%$ reduction in the tension of the messenger cable on the equilibrium configuration of the catenary (the black (dark) line: before the defect; the red (grey) line: after the defect).

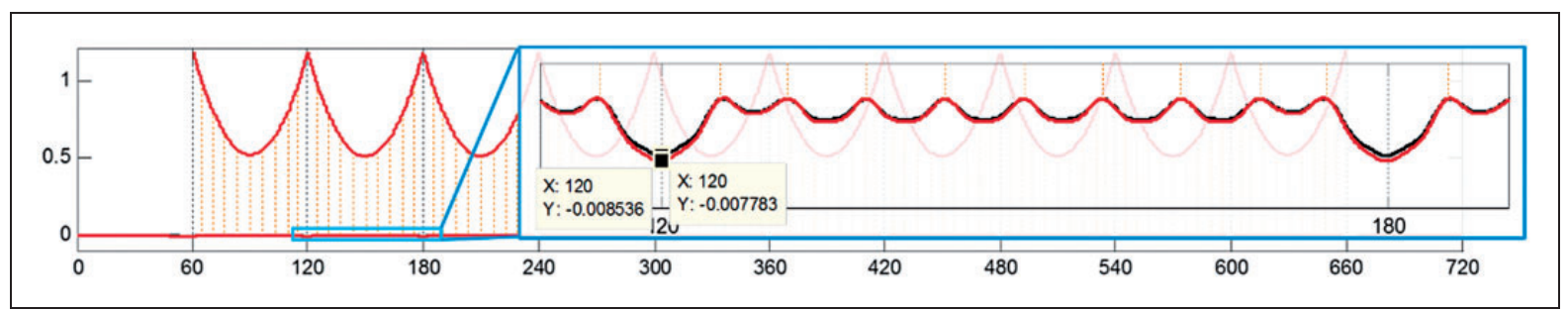

Figure 9. The effect of $10 \%$ reduction in the tension in the contact wire on the equilibrium configuration of the catenary (the black (dark) line: before the defect; the red (grey) line: after the defect).

Table 2. A comparison between the static force $(\mathrm{N})$ of droppers in various FEM or FDM software programs, and the predictions by the exact analytical method of this research for the French LN2 or the Italian C270 systems.

\begin{tabular}{lcllllll}
\hline SW name & $\begin{array}{c}\text { Drop. I, } \\
\mathrm{x}=4.50\end{array}$ & $\begin{array}{l}\text { Drop. 2, } \\
\mathrm{x}=10.25\end{array}$ & $\begin{array}{l}\text { Drop. 3, } \\
\mathrm{x}=16.00\end{array}$ & $\begin{array}{l}\text { Drop. 4, } \\
\mathrm{x}=21.75\end{array}$ & $\begin{array}{l}\text { Drop. 5, } \\
\mathrm{x}=27.5\end{array}$ & $\begin{array}{l}\text { Total force of } \\
\text { all droppers }\end{array}$ & $\begin{array}{l}\text { \% Error (weight of } \\
\text { contact wire) }\end{array}$ \\
\hline PrOSA & - & - & - & - & - & - & - \\
PantoCat & 169.45 & 49.14 & 55.44 & 47.38 & 55.42 & 698.24 & -7.213 \\
SPOPS & 198.10 & 53.06 & 54.24 & 54.17 & 54.15 & 773.29 & 2.760 \\
CaPaSIM & 161.98 & 52.14 & 51.90 & 51.98 & 51.69 & 687.69 & -8.615 \\
PCaDA & 195.57 & 52.15 & 52.10 & 52.08 & 52.06 & 755.86 & 0.444 \\
Gasen-do & 171.32 & 50.09 & 55.89 & 47.80 & 55.84 & 706.04 & -6.177 \\
OSCAR & 167.90 & 50.67 & 55.47 & 47.52 & 55.39 & 698.51 & -7.177 \\
PCRUN & 162.61 & 52.43 & 56.20 & 48.55 & 56.20 & 695.78 & -7.540 \\
CANDY & 155.56 & 52.24 & 56.58 & 48.01 & 54.80 & 679.58 & -9.693 \\
PACDIN & 164.14 & 50.30 & 55.30 & 47.34 & 55.29 & 689.45 & -8.381 \\
Standard deviation & 14.067 & 1.255 & 1.614 & 2.442 & 1.527 & 30.621 & - \\
This research (exact & 197.73 & 47.40 & 55.74 & 47.25 & 55.81 & 752.1 & 0.057 \\
analytical method) & & & & & & & \\
\hline
\end{tabular}

SW: software.

effect of $10 \%$ reduction in the tension of the contact wire is, generally, insignificant. When the objective profile of the contact wire is zero, changing the tension in the contact wire cannot have a significant effect on the equilibrium configuration of the contact wire compared with the tension loss in the messenger cable.
Using this method, a combination of different defects, such as the tension loss in both the messenger cable and the contact wire or the breaking of the two droppers at the same time, can be studied as well. In addition, the effect of the added mass on the contact wire or the messenger cable can be considered. 
Table 3. Deviation of the contact wire from its initial position at the location of the droppers after $5 \%$ reduction in the messenger wire tension.

\begin{tabular}{lcllll}
\hline & Dropper I & Dropper 2 & Dropper 3 & Dropper 4 & Dropper 5 \\
\hline Field measurement $(\mathrm{mm})$ & 9.5 & 13.7 & 17.5 & 19.7 & 20.1 \\
This research $(\mathrm{mm})$ & 10.1 & 14.2 & 17.8 & 20.1 & 20.5 \\
\hline
\end{tabular}

\section{Validation}

For validation purposes, the calculated static force of the droppers, for the example case is compared with the results from some other software. The data in Table 2 are extracted from Facchinetti and Bruni $^{2}$ that presented different software calculations for the static force of each dropper for the French LN2 or the Italian C270 systems. ${ }^{1}$ The last row in Table 2 is the result of this research for the dropper force calculations, for the same French LN2 or the Italian C270 systems, that is based on the exact analytical method.

The results prove that the error in this method is insignificant in comparison with the other methods. In fact, based on the actual measurements, the whole weight of the contact wire and its attached clamps in 10 spans is $7525.21 \mathrm{~N}$ and the summation of the dropper force is $7520.89 \mathrm{~N}$. The small error exists due to the weight of the contact wire carried by the support of the contact wire at both sides of the section.

In order to evaluate the accuracy of the results that are achieved from the analytical model, a field test is organized. In this test, the tension of the messenger cable is reduced by removing the weights of the tension wheel. The distance between the contact wire at the dropper clamp position and the track centerline is measured using a laser measuring device with a precision of $0.1 \mathrm{~mm}$. The measured distances are compared with the initial distance, that is, the distance before reducing the tension. The deviation from the initial position is reported in Table 3 .

Since any variation in the shape of the dropper due to the breakage of one dropper or reduction of tension in the contact wire is insignificant, the field measurement for such case is ignored. The calculated and the measured data that are presented in Table 3 prove that the proposed analytical method of this research can properly estimate the shape of the catenary. The degree of precision in the calculations is a credit to the modeling procedure.

\section{Conclusions}

In this article, an exact analytical solution for formfinding of normal and defective catenaries is proposed. For normal catenaries, the dead load or the static force in the droppers is calculated such that the contact wire can be in its objective profile. Since the vertical offset between the contact wire and the messenger cable does not change during the defect, the equilibrium configuration of the catenary can be achieved by recalculating the system equations. The proposed method does not include any iterative procedure. It is fast and accurate. This method can be used within any other simulation model (FEM or FDM) since the output of this method is the droppers' dead loads. The results of catenary form-finding after incurring any of the three common defects show that, for example, breaking of a dropper can create sag, twice as big as the biggest possible sag in a normal catenary. In addition, the shape of the catenary is more sensitive to the varying tension in the messenger cable compared to the contact wire.

\section{Declaration of Conflicting Interests}

The author(s) declared no potential conflicts of interest with respect to the research, authorship, and/or publication of this article.

\section{Funding}

The author(s) disclosed receipt of the following financial support for the research, authorship, and/or publication of this article: This research was supported by the office for "National Master Plan for High Speed Trains" at Iran University of Science and Technology. The authors are grateful for the support awarded.

\section{ORCID iD}

Mohammad Ali Rezvani (D) http://orcid.org/0000-00031819-7772

Habibolah Molatefi (D) http://orcid.org/0000-0002-75849637

\section{References}

1. Finner L, Poetsch G, Sarnes B, et al. Program for catenary-pantograph analysis, PrOSA statement of methods and validation according EN 50318. Veh Syst Dyn 2015; 53: 305-313.

2. Facchinetti A and Bruni S. Special issue on the pantograph-catenary interaction benchmark. Veh Syst Dyn 2015; 53: 303-304.

3. Ambrósio J, Pombo J, Antunes P, et al. PantoCat statement of method. Veh Syst Dyn 2015; 53: 314-328.

4. Cho YH. SPOPS statement of methods. Veh Syst Dyn 2015; 53: 329-340.

5. Lewis WJ. Tension structures: form and behaviour. London: Thomas Telford, 2003.

6. Veenendaal D and Block P. An overview and comparison of structural form finding methods for general networks. Int J Solids Struct 2012; 49: 3741-3753.

7. Lopez-Garcia O, Carnicero $\mathrm{A}$ and Torres V. Computation of the initial equilibrium of railway overheads based on the catenary equation. Eng Struct 2006; 28: $1387-1394$. 
8. Such M, Jimenez-Octavio JR, Carnicero A, et al. An approach based on the catenary equation to deal with static analysis of three dimensional cable structures. Eng Struct 2009; 31: 2162-2170.

9. Cho YH, Lee K, Park Y, et al. Influence of contact wire pre-sag on the dynamics of pantograph-railway catenary. Int J Mech Sci 2010; 52: 1471-1490.

10. Jönsson P-A, Stichel S and Nilsson C. CaPaSIM statement of methods. Veh Syst Dyn 2015; 53: 341-346.

11. Zhou N, Lv Q, Yang Y, et al. TPL-PCRUN Statement of methods. Veh Syst Dyn 2015; 53: 380-391.

12. Tur M, García E, Baeza L, et al. A 3D absolute nodal coordinate finite element model to compute the initial configuration of a railway catenary. Eng Struct 2014; 71: 234-243.

13. Massat J-P, Balmes E, Bianchi J-P, et al. OSCAR statement of methods. Veh Syst Dyn 2015; 53: 370-379.

14. Yang C, Zhang W, Zhang J, et al. Static form-finding analysis of a railway catenary using a dynamic equilibrium method based on flexible multibody system formulation with absolute nodal coordinates and controls. Multibody Syst Dyn 2017; 39: 221-247.

15. Song D, Jiang Y and Zhang W. Dynamic performance of a pantograph-catenary system with consideration of the contact surface. Proc IMechE, Part F: J Rail and Rapid Transit 2018; 232: 262-274.

16. Gregori S, Tur M, Nadal E, et al. An approach to geometric optimisation of railway catenaries. Veh Syst Dyn 2018; 56: 1162-1186.

17. EN 50318:2002. Railway applications - Current collection systems - Validation of simulation of the dynamic interaction between pantograph and overhead contact line; 14-16.

\begin{tabular}{|c|c|}
\hline$F_{d r i}$ & $i$ th dropper force \\
\hline$F_{g}$ & weight load \\
\hline$\vec{H}$ & Encumbrance \\
\hline$k_{d r i}$ & stiffness of $i$ th dropper \\
\hline$k_{r i}$ & stiffness of $i$ th registration arm \\
\hline$k_{s i}$ & stiffness of $i$ th support \\
\hline$\left[K_{c w}\right]$ & stiffness matrix of contact wire \\
\hline$\left[K_{m c}\right]$ & stiffness matrix of messenger cable \\
\hline $\overrightarrow{M_{m}}$ & $\begin{array}{l}\text { mass of messenger cable at dropper } \\
\text { connection points }\end{array}$ \\
\hline $\overrightarrow{M_{c}}$ & $\begin{array}{l}\text { mass of contact wire at dropper con- } \\
\text { nection points }\end{array}$ \\
\hline$T_{m c}$ & tension of messenger cable \\
\hline$T_{c w}$ & tension of contact wire \\
\hline $\overrightarrow{w_{g c w}}$ & $\begin{array}{l}\text { vertical deflection of contact wire due } \\
\text { to gravity at dropper connection points }\end{array}$ \\
\hline $\overrightarrow{w_{o c w}}$ & $\begin{array}{l}\text { objective profile of contact wire at } \\
\text { dropper connection points }\end{array}$ \\
\hline $\overrightarrow{y_{1}}$ & $\begin{array}{l}\text { vertical deflection of the dropper con- } \\
\text { nection points on the messenger cable } \\
\text { measured from the reference axes of the } \\
\text { messenger cable }\end{array}$ \\
\hline $\overrightarrow{y_{2}}$ & $\begin{array}{l}\text { vertical deflection of the dropper con- } \\
\text { nection points on the contact wire } \\
\text { measured from the reference axes of the } \\
\text { messenger cable }\end{array}$ \\
\hline $\overrightarrow{y_{0}}$ & $\begin{array}{l}\text { objective position of the dropper con- } \\
\text { nection points on the contact wire } \\
\text { measured from the reference axes of the } \\
\text { contact wire }\end{array}$ \\
\hline$\rho A$ & mass per unit length of wire \\
\hline
\end{tabular}

\section{Appendix}

\section{Notation}

EI

bending stiffness 